IP Periodica Polytechnica Electrical Engineering and Computer Science

61(2), pp. 217-223, 2017

https://doi.org/10.3311/PPee.9742

Creative Commons Attribution (1)

RESEARCH ARTICLE

\section{Constructions for the Optimal Pebbling of Grids}

\author{
Ervin Győri1 ${ }^{1,2}$, Gyula Y. Katona ${ }^{3,4^{*}}$, László F. Papp ${ }^{3}$
}

Received 11 July 2016; accepted after revision 19 April 2017

\begin{abstract}
In [6] the authors conjecture that if every vertex of an infinite square grid is reachable from a pebble distribution, then the covering ratio of this distribution is at most 3.25. First we present such a distribution with covering ratio 3.5, disproving the conjecture. The authors in the above paper also claim to prove that the covering ratio of any pebble distribution is at most 6.75. The proof contains some errors. We present a few interesting pebble distributions that this proof does not seem to cover and highlight some other difficulties of this topic.
\end{abstract}

\section{Keywords}

optimal pebbling, pebbling, grid graph

\footnotetext{
${ }^{1}$ Alfréd Rényi Institute of Mathematics, Budapest, Hungary

${ }^{2}$ Department of Mathematics, Central European University, Budapest, Hungary

${ }^{3}$ Department of Computer Science and Information Theory, Faculty of Electrical Engineering and Informatics, Budapest University of Technology and Economics, 1521 Budapest, P.O. Box 91, Hungary

${ }^{4}$ MTA-ELTE Numerical Analysis and Large Networks Research Group, Hungary

*Corresponding author, e-mail: kiskat@cs.bme.hu
}

\section{Introduction}

Graph pebbling has its origin in number theory. It is a model for the transportation of resources. Starting with a pebble distribution on the vertices of a simple connected graph, a pebbling move removes two pebbles from a vertex and adds one pebble at an adjacent vertex. We can think of the pebbles as fuel containers. Then the loss of the pebble during a move is the cost of transportation. A vertex is called reachable if a pebble can be moved to that vertex using pebbling moves. There are several questions we can ask about pebbling. One of them is: How can we place the smallest number of pebbles such that every vertex is reachable? The minimum number of pebbles in such a pebble distribution is called the optimal pebbling number of the graph. The optimal covering ratio of a graph is the number of vertices of the graph divided by the optimal pebbling number. Moreover, the covering ratio of an arbitrary distribution is the number of vertices reachable from the distribution divided by the number of pebbles in the distribution. For a comprehensive list of references for the extensive literature see the survey papers $[3,4,5]$.

In Section 3 we show a pebble distribution which disproves a conjecture of Xue and Yerger. Section 4 and 5 contain some interesting counterexamples for some lemmas stated in [6]. We also mention some phenomenons why we think that the proof of Theorem 8 of [6] can not be corrected, and a proper proof requires a different approach.

In the last section we introduce a new problem, called optimal integer fractional covering ratio, where the tools introduced in [6] can be used. We give a lower and an upper bound on the optimal integer fractional covering ratio of large grids.

\section{Definitions}

In this section we summarize the definitions which the paper uses. We start with basic ones, which are well known in the area of pebbling, then continue with more complicated ones, which were introduced in [6]. 


\subsection{Traditional pebbling}

A pebbling distribution $D$ is a $V(G) \rightarrow \mathbb{N}$ function. If $D(v) \geq 2$ and $u$ and $v$ are adjacent vertices, then we can apply a $(v \rightarrow u)$ pebbling move. It decreases $D(v)$ by two and increases $D(u)$ by one. A vertex $v$ is reachable under $D$ if either $D(v) \geq 1$ or we can apply a sequence of pebbling moves such that the last one is an $(u \rightarrow v)$ move.

A distribution is solvable if each vertex is reachable under it. We use $|D|$ for the size of distribution $D$, which is the total number of pebbles placed on the graph. A distribution is optimal on graph $G$ if its size is minimal among all solvable distributions of $G$. The optimal pebbling number is the size of an optimal distribution and it is denoted by $\pi_{\mathrm{opt}}(G)$.

The coverage of distribution $D$ is the set of reachable vertices. We denote the size of this set by $\operatorname{Cov}(D)$. The covering ratio of $D$ is defined as $\frac{\operatorname{Cov}(D)}{|D|}$. The covering ratio of an optimal distribution of $G$ is called as the optimal covering ratio of $G$.

\subsection{Infinite graphs}

We are mainly interested in the pebbling parameters of square grids. We denote the $n \times n$ square grid by $G_{n \times n}$ and we write $G_{\text {inf }}$ for the infinite square grid.

In [6] the authors talk about $G_{\text {inf }}$, they do not provide a proper definition for the covering ratio in the case when the distribution is infinite. In their reasoning they assume that the number of pebbles is finite, therefore we will assume the same. When solvability comes into play, we think about arbitrarily large but finite square grids, whose border's size is marginal compared to their total size. Therefore their covering ratio is well defined. We define the optimal covering ratio of $G_{i n f 1}$ as the limit of larger and larger square grids's optimal covering ratios. Conversely, it can be written as $\lim _{n \rightarrow \infty} \frac{\left|G_{n x x}\right|}{\pi_{\text {op }}\left(G_{n \times n}\right)}$.

Proposition 1. $\lim _{n \rightarrow \infty} \frac{\left|G_{n \times n}\right|}{\pi_{\text {opt }}\left(G_{n \times n}\right)}$ exists.

Proof: We prove that the $A-\frac{\pi_{\text {or }}\left(G_{\text {nox }}\right)}{n^{2}}<\epsilon$ sequence is convergent. It is easy to prove that $\pi_{\mathrm{opt}}\left(G_{n \times n}\right) \geq \frac{1}{9} n^{2}$, moreover Theorem 2 implies a better lower bound. Therefore the limit of this series is not zero which implies the proposition.

Let $\epsilon$ be an arbitrary positive real number and $A=\liminf _{n \rightarrow \infty} \frac{\pi_{\text {opt }}\left(G_{n \times n}\right)}{n^{2}}$. Choose $m$ large enough such that $\frac{6}{m}<\epsilon, \frac{\pi_{\text {opt }}\left(G_{m \times m}\right)}{m^{2}}-A \leq \frac{\epsilon}{2}$ and for every $n \geq m$ the inequality $A-\frac{\pi_{\text {opt }}\left(G_{\text {nxn }}\right)}{n^{2}}<\epsilon$ holds. We show that $n \geq m^{2}$ implies $\left|A-\frac{\pi_{\text {opt }}\left(G_{n \times n}\right)}{n^{2}}\right|<\epsilon$.
Write $n$ as $k m+r$ such that $0 \leq r<m$ and partition $G_{n \times n}$ into $k^{2}$ pieces of disjoint $G_{m \times m}$ and $r^{2}+2 r k m$ remaining vertices. We use its optimal distribution on each $G_{m \times m}$ and place one pebble on each remaining vertex. In such a way we obtain a solvable distribution of $G_{n \times n}$. Therefore:

$$
\begin{aligned}
&-\epsilon<\frac{\pi_{\mathrm{opt}}\left(G_{n \times n}\right)}{n^{2}}-A \leq \frac{k^{2} \pi_{\mathrm{opt}}\left(G_{m \times m}\right)+r^{2}+2 r k m}{k^{2} m^{2}+r^{2}+2 r k m}-A \\
& \leq \frac{k^{2} \pi_{\mathrm{opt}}\left(G_{m \times m}\right)+r^{2}+2 r k m}{k^{2} m^{2}}-A \\
& \leq \frac{\pi_{\mathrm{opt}}\left(G_{m \times m}\right)}{m^{2}}+\frac{r^{2}}{m^{4}}+\frac{2 r}{m^{2}}-A \leq \frac{\pi_{\mathrm{opt}}\left(G_{m \times m}\right)}{m^{2}}-A+\frac{3}{m}<\frac{\epsilon}{2}+\frac{\epsilon}{2}=\epsilon
\end{aligned}
$$

We note that there are several ways to define pebbling parameters for infinite graphs by considering infinite distributions. Nevertheless, it is beyond the scope of this paper.

\subsection{Combining distributions}

Assume that we have two distributions $D$ and $D^{\prime}$. We say that these two distributions interact at vertex $v$ if $v$ is reachable under both. Vertex $v$ is a boundary vertex of $D$ if $v$ is reachable under $D$ but one of its neighbours is not.

It is a natural idea to unify two distributions $D$ and $D^{*}$ by placing $D(v)+D^{*}(v)$ pebbles at $v$, to create a bigger one. $D$ and $D^{*}$ are stronger together in the sense, that some vertices are not reachable under $D$ nor $D^{*}$, but they are reachable under the $D^{\prime}$ which we get by combining them. This phenomenon requires the presence of interacting vertices.

For example, if an interaction vertex is boundary in both $D$ and $D^{*}$ and one of its neighbours is not reachable under $D$ and $D^{*}$, then this neighbour is reachable under $D^{\prime}$.

A unit is a vertex having at least one pebble. A unit distribution contains only one unit.

Using the combination method we can build any distribution from unit distributions. It often happens, that the coverage of a unit is disjoint from coverage of the rest of the distribution. So the unit and the rest do not share an interaction vertex and they can be handled separately. We say that these units are lonely.

We can also ask that what is the difference between the covering ratios of $D$ and $D^{\prime}$. Of course, it depends on $D^{*}$, but we would like to measure it. This motivates the definition of marginal covering ratio, which is the following:

$$
\frac{\operatorname{Cov}\left(D^{\prime}\right)-\operatorname{Cov}(D)}{\left|D^{\prime}\right|-|D|}
$$

We can not compute the covering ratio of $D^{\prime}$ if we know the covering ratio of $D$ and the marginal covering ratio. On the other hand, we can state upper bounds, which we are interested in. 


\subsection{Fractional pebbling}

A variation of the pebbling problem if we allow fractional pebbles. This leads to the area of fractional pebbling, which is well studied in [2].

A continuous distribution on $G$ is a $V(G) \rightarrow[0, \infty)$ function. A continuous pebbling move removes $t$ pebbles from a vertex and place $t / 2$ pebbles to an adjacent vertex, where $t$ can be any positive real number.

The optimal fractional pebbling number is the size of the smallest solvable continuous distribution. It can be calculated by solving a linear program and it is a lower bound on the optimal pebbling number.

Let $D$ be a continuous distribution. The weight function of $D$, which is defined on the vertex set of $G$, is defined as:

$$
W_{D}(u)=\sum_{v \in V(G)} D(v) 2^{-d(u, v)}
$$

It tells us the number of pebbles that can be moved to a vertex under $D$ by continuous pebbling moves. It is a very useful tool, widely used by several authors in this topic.

In a solvable distribution the weight of each vertex is at least one. However, if we are considering optimal pebbling distributions, then several vertices have more weight than one. We can consider this extra weight as an excess, and try to calculate it. The sum of these values gives an estimate on the difference between the fractional and the traditional optimal pebbling numbers.

So we define the excess weight function as:

$$
\widehat{W}_{D}(u)=\left\{\begin{array}{cc}
W_{D}(u)-1 & \text { if } W_{D}(u)>1, \\
W_{D}(u) & \text { if } W_{D}(u) \leq 1 .
\end{array}\right.
$$

With the help of this function, we can give a fractional generalization of covering ratio. To construct the numerator we count the number of reachable vertices and also add the weight of not reachable vertices. The covering ratio ceiling of $D$ is:

$$
\frac{\sum_{v \in V(G)} W_{D}(v)-\sum_{v \in V(G)} \widehat{W}_{D}(v)}{\sum_{v \in V(G)} D(v)} .
$$

Like the marginal covering ratio we also define a quantity which measures in some way the change of covering ratio ceiling in case of adding some extra pebbles. So let $D$ and $D^{\prime}$ be distributions, such that $D(v) \leq D^{\prime}(v)$ for each vertex. The marginal covering ratio ceiling of these distributions is defined by

$$
\frac{\left(\sum_{v \in V(G)} W_{D}(v)-\sum_{v \in V(G)} \widehat{W}_{D}(v)\right)-\left(\sum_{v \in V(G)} W_{D}(v)-\sum_{v \in V(G)} \widehat{W}_{D}(v)\right)}{\sum_{v \in V(G)} D^{\prime}(v)-\sum_{v \in V(G)} D(v)} .
$$

\section{Lower bound for the optimal covering ratio of the grid}

Conjecture 2 in [6] states that if every vertex of an infinite square grid is reachable from a pebble distribution, then the covering ratio of this distribution is at most 3.25.

We present a sequence of distributions on big grids whose covering ratios converge to 3.5 , disproving the conjecture. Repeating periodically the optimal distribution of $G_{n \times n}$ results a solvable distribution on the infinite grid. Therefore considering real infinite graphs and distributions can not decrease the optimal covering ratio, if it is defined intuitively.

A distribution is shown on Fig. 1, units consisting of four pebbles are placed to every other vertex on every 7 th diagonal. It is easy to calculate that the covering ratios of such distributions tends to $7 / 2=3.5$. The arrows and the shaded areas on the figure indicate how to reach all vertices of the grid from this distribution. We conjecture that this is best possible.

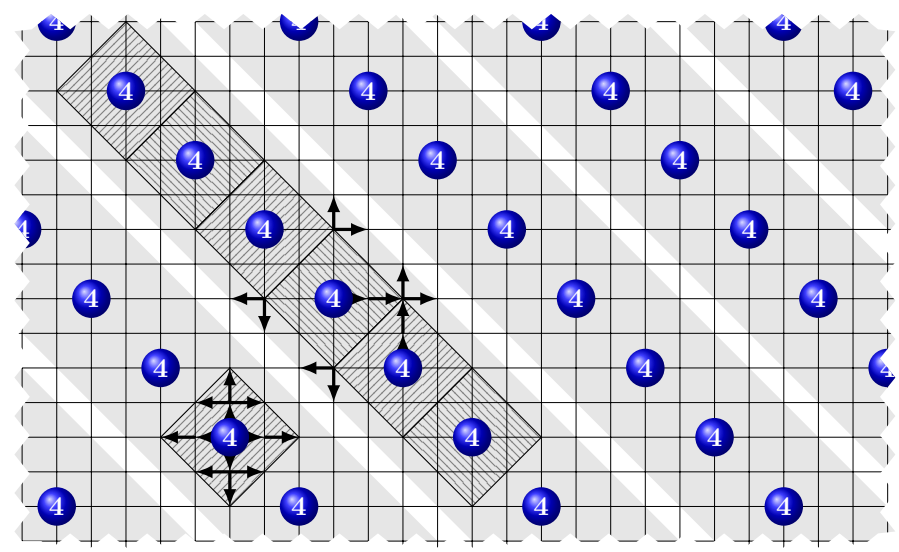

Fig. 1 Pebble distribution of the grid with covering ratio 3.5

Conjecture 1. If every vertex of an infinite square grid is reachable from a pebble distribution, then the covering ratio of this distribution is at most 3.5 .

\section{Comments on the upper bound for the optimal covering ratio of the grid}

In [6] the authors claim to prove that the optimal covering ratio of the grid is at most 6.75 (see [6]). The proof contains some errors. Although some of these errors may be corrected somehow, in our opinion some others cannot be corrected. We do not see how to complete the proof, but we believe that the statement is true. In forthcoming paper [1] we are to prove a better bound: the covering ratio is at most 6.5 . In the rest of this note we point out some errors in the proof and show some interesting pebble distributions that highlight the difficulties of this problem.

First we summarize the above mentioned proof. It is an inductive proof on the number of units contained in the distribution. First, as the base case, it is shown that the theorem holds for one unit. Now assume that it also holds for any distribution containing $n$ units. Consider a distribution of $n+1$ 
units, remove an arbitrary unit, apply the inductive hypothesis for the remaining units. Depending on the position of the removed unit and the remaining distribution apply [6] or [6] to complete the proof. (In the original paper in Section 6 all reference to the lemmas are shifted by one, so any reference to Lemma $i$ should be to Lemma $i-1$.)

\subsection{Comments on [6]}

The lemma states: For initial distribution $D$ and unit $U$, if only the boundary vertices of $U$ are reachable via pebbles from $D$, then the marginal covering ratio of a unit on $G_{i n f}$ is at most 4.25 .

We present several counterexamples to this lemma.

In the first example (see Fig. 2) let $D$ be the distribution consisting units of size 1 in a horizontal row. The covering ratio of $D$ is clearly 1 . Now let $U$ be a unit of size 2 placed at the end of this row. Only a boundary vertex of $U$ is reachable from $D$ (i.e. a unit of $D$ is on the boundary of $U$ ), so the conditions of the lemma are satisfied. However after adding $U$, all vertices in the shaded area become reachable. Since the size of $U$ is constant (it is 2), the marginal covering ratio depends on the size of $D$. It can be arbitrary large even if we consider finite $D$ distributions, and it can be infinite if $D$ is made infinitely large.

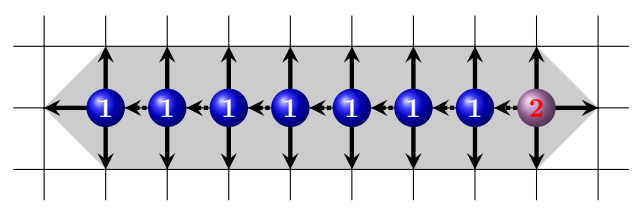

Fig. 2 Distribution with large marginal covering ratio.

Another example is shown on Fig. 3, where a similar problem appears. Adding a unit of size 1 can increase the number of newly reachable vertices by an arbitrary large amount.

The proof presented in [6] works only for the following weaker statement:

Lemma 1. Let $D$ be a distribution and $U$ be a unit such that only the boundary vertices of $D$ and $U$ interact. Assume, moreover, that if $D$ has a unit of size one, then $U$ also has size one. Then, the marginal covering ratio of $U$ on $G_{\text {inf }}$ is at most 4.25 .

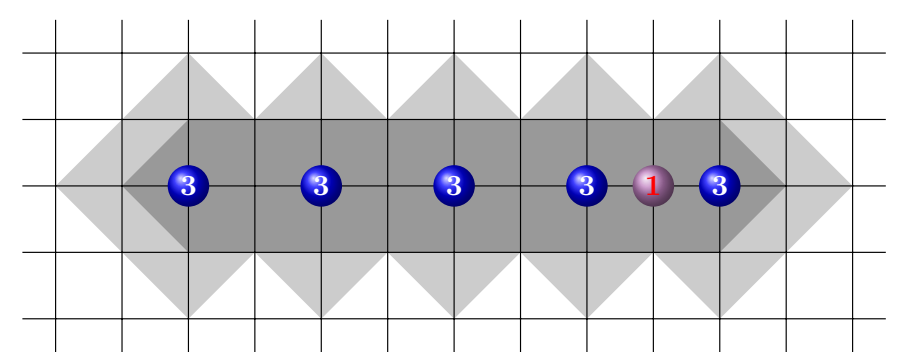

Fig. 3 Distribution with large marginal covering ratio.

\subsection{Comments on the inductive step}

In the inductive step, we assume that there is a distribution $D$ with covering ratio at most 6.75 . Now we add a unit $U$ . The authors do not explain this step in detail, but we assume that their intention is to apply [6] if "only the boundary vertices of $U$ are reachable via pebbles from $D$ ", and apply [6] if "the unit interacts not only on the boundary vertices".

The examples in the previous subsection show that in some cases, when some units of size 1 are involved, [6] cannot be used. We think that the authors intended to handle this problem with the following sentence in the proof of [6]: "We assume that $D$ does not contain lonely units with one pebble because if $D$ contains those units, we can remove them first and add them after $U$ has been added." This suggests that in the inductive step one should be more careful how to select the unit to be removed, remove the lonely units first. The above example suggests that this does not work. On the other hand, it looks promising to always remove a unit which is on the "boundary" of $D$, but now the boundary is understood differently, something like the "convex hull". However, this approach does not look easy.

Now let us consider the case when [6] is applied. (The lemma in fact states, that the marginal covering ratio ceiling in this case is at most 6 , however, the proof gives 6.75 , so this is clearly a typo). We give an example, when this fails to prove the inductive step: Two units of size 2 on adjacent vertices of the grid. In this case one of the units is $D$ the other is $U$, the interaction happens not only on the boundaries. The covering ratio of $D$ is $2.5<6.75$, so the inductive hypothesis holds. Now [6] implies that the marginal covering ratio ceiling of $U$ is at most 6.75 . These facts do not imply that the covering ratio of $D \cup U$ is at most 6.75. (Of course, the covering ratio is in fact $2<6.75$, just the proof does not imply this.)

The covering ratio is $\operatorname{Cov}(D) /|D|$. However, in the definition of the marginal covering ratio ceiling neither $\operatorname{Cov}(D)$ nor, more importantly, $\operatorname{Cov}(D \cup U)$ appear, so it seems impossible that these two inequalities would imply anything useful for $\operatorname{Cov}(D \cup U)$. We suspect that the intention of the authors was to say that if the marginal covering ratio ceiling of $D$ is at most 6.75 and the marginal covering ratio ceiling of the pair $(D, U)$ is at most 6.75 , then the covering ratio ceiling of $D \cup U$ is at most 6.75. This implication is correct, but then we do not see how to obtain the desired bound for the covering ratio. Of course, since the covering ratio ceiling is an upper bound for the covering ratio, it would be enough to prove with the previous argument that the covering ratio ceiling is at most 6.75 . The above argument does not give this. The covering ratio ceiling of $D \cup U$ is in fact 7.25 , implying only that the covering ratio is at most 7.25 . The basic problem is that the covering ratio ceiling of the only unit of size 2 in $D$ is 8.5 . 


\section{Connection between the covering ratio and the covering ratio ceiling}

The covering ratio ceiling is an upper bound for the covering ratio. It is also clear that the marginal covering ratio ceiling cannot be large if we add a new unit to a distribution. Does this imply something about the change in the covering ratio?

Theorem 1. The distribution given in Fig. 4 shows that the covering ratio can increase by more than one while the covering ratio ceiling decreases.

Proof: Let us consider the distribution in Fig. 4. A unit of size 3 on every second vertex in a row of length $2 n+1(n+1$ such units in a row), repeated in every fifth row (see Fig. 4) in $5 m+1$ rows. The marginal covering ratio ceiling of a unit is $\frac{\Delta W-\Delta \widehat{W}}{\Delta|D|}$, where $\Delta W$ is the sum of the changes of the weights which all pebbles contributes to a vertex, $\Delta \widehat{W}$ is the same for the weight ceiling function, and $\Delta|D|$ is the number of added pebbles.

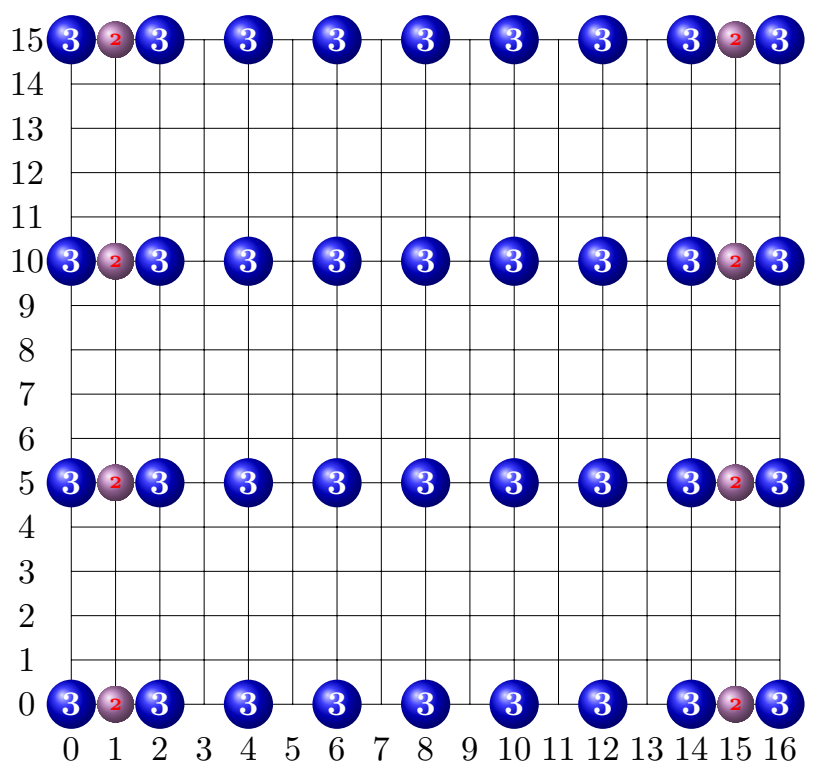

Fig. 4 Pebble distribution with increasing covering ratio.

Let us first see the weights at all vertices in this distribution. For example the total weight for every vertex in row 0 is clearly at least 3 . Thus the total weight at every vertex in row 1 is at least $3 / 2$. The weight for every vertex in row 2 is at least $3 / 4+3 / 8=9 / 8$, since the pebbles in row 0 contribute at least $3 / 4$ and pebbles in row 5 contribute at least $3 / 8$ to its weight. The same is true for all other rows, therefore it is clear that the weight of every vertex is at least 1 .

If the weight of a vertex is at least 1 then any positive change in the total weight will result in the same amount of positive change of $\widehat{W}$. So these vertices contribute 0 to $\Delta W-\Delta \widehat{W}$. This implies that if further units are added to this distribution, then their marginal covering ratio ceiling will always be 0 . This calculation also shows that the covering ratio ceiling is

$$
\frac{(5 m+1)(2 n+1)}{3(n+1)(m+1)}<\frac{10}{3}
$$

Let us calculate the covering ratio of the distribution. It is easy to see that each vertex in rows $0,1,4,5,6,9,10,11, \ldots$ is reachable, but no vertex in rows $2,3,7,8, \ldots$ is reachable. Therefore, the covering ratio is

$$
\frac{(3 m+1)(2 n+1)}{3(n+1)(m+1)}<2 .
$$

Now we add units of size 2 near both ends of the row containing pebbles (the lighter, smaller pebbles on Fig. 4) one by one. The above argument shows that the marginal covering ratio ceiling is 0 in every step, and the covering ratio ceiling becomes

$$
\frac{(5 m+1)(2 n+1)}{3(n+1)(m+1)+4 m}<\frac{(5 m+1)(2 n+1)}{3(n+1)(m+1)}<\frac{10}{3},
$$

so it is decreasing. On the other hand, it is easy to see that one can move 4 pebbles to any vertex of row $5 k$, thus every vertex of the grid becomes reachable. Therefore the covering ratio is also

$$
\frac{(5 m+1)(2 n+1)}{3(n+1)(m+1)+4 m}
$$

which is close to $\frac{10}{3}$ if $n$ and $m$ are large enough. So while the covering ratio ceiling is decreasing, the covering ratio is increasing from 2 to $\frac{10}{3}$.

\section{Optimal fractional covering ratio of integer distributions}

The above arguments lead to an interesting question. What is the best upper bound we can hope for using [6]? The idea behind this lemma is that the fractional covering ratio is an upper bound on the covering ratio, and if the starting pebble distribution has only integer number of pebbles, then there must be a certain amount of excess weight. It is easy to prove that the optimal fractional covering ratio on the grid is 9 . The optimal distribution is obtained by placing $1 / 9$ pebbles at every vertex. How does this change if we only consider integer distributions? Let us call this the optimal integer fractional covering ratio, and denote it by $\operatorname{IFCov}(G)$. We give upper and lower bounds for this ratio in case of the $n \times n$ grids .

Theorem 2. For any $\varepsilon>0$ there exists $\mathrm{n}(\varepsilon)$ such that if $\mathrm{n}>$ $\mathrm{n}(\varepsilon)$ then

$$
7-\varepsilon \leq \operatorname{IFCov}\left(G_{n \times n}\right) \leq \frac{213}{25}=8.52
$$

Proof: Consider the distribution given in Fig. 5. It is easy to calculate, that the number of pebbles is $n^{2} / 7+O(n)$, therefore if $n$ is large enough, then $\operatorname{IFCov}\left(G_{n \times n}\right) \geq 7-\varepsilon$. We need to show, that every vertex of the grid is covered by this 
distribution in the fractional sense, i.e. the weight of each vertex is at least one. This is clearly true for the vertices, where a pebble is placed. By the structure of the distribution, it is clear that it is enough to show this for the vertices marked with $A, B, C$. For $A$ : there is 1 pebble at distance 1,1 at distance 2, 1 at distance 3 and 3 at distance 4 . Thus the weight at $A$ is at least $\frac{1}{2}+\frac{1}{4}+\frac{1}{8}+\frac{3}{16}>1$. Similar calculations show that the weight at $B$ is at least $\frac{2}{4}+\frac{3}{8}+\frac{1}{16}+\frac{2}{32}=1$, and the weight at $C$ is at least $\frac{1}{2}+\frac{1}{4}+\frac{1}{8}+\frac{3}{16}>1$. For vertices near the border, the extra pebbles placed on the border guarantees the required weight. Hence the lower bound is proven.

To prove the upper bound, the following Lemma is needed.

Lemma 2. If a distribution covers all vertices of the grid in the fractional sense and the vertex $v$ contains a unit of size $k$ then the excess weight at this vertex is at least $\frac{12}{25} k$.

Proof: If $k>1$ then the excess weight is at least $k-1>\frac{12}{25} k$, so the claim clearly holds. Therefore we only have to deal with the $k=1$ case. The contribution of this pebble to the weight of its neighbours is $\frac{1}{2}$, thus the contribution of other pebbles must also be at least $\frac{1}{2}$. Similarly, the contribution of this pebble is $\frac{1}{4}$ to the vertices at distance 2 from $v$, thus the contribution of other pebbles must also be at least $\frac{3}{4}$. So consider the distance 2 neighborhood of $v$ and partition all vertices of the grid according to Fig. 6. For simplicity denote by $x_{i}\left(y_{i}\right)$ the total weight contribution of all pebbles in region $X_{i}\left(Y_{i}\right)$ to the corresponding vertex. Consider now for example vertex $x_{1}$. The weight from pebbles in $X_{1}$ is $x_{1}$, the weight from pebbles in $Y_{1}$ is clearly $\frac{1}{2} y_{1}$, since the distance of any pebble in $Y_{1}$ and $x_{1}$ is one more than the distance to $y_{1}$. Similarly, the weight from $X_{2}$ to $x_{1}$ is $\frac{1}{4} x_{2}$, from $Y_{2}$ to $x_{1}$ is $\frac{1}{8} y_{2}$, etc. Since the total weight at $x_{1}$ must be at least 1 and the contribution of $v$ is $\frac{1}{2}$,

$$
1 \leq \frac{1}{2}+x_{1}+\frac{1}{4} x_{2}+\frac{1}{4} x_{3}+\frac{1}{4} x_{4}+\frac{1}{2} y_{1}+\frac{1}{8} y_{2}+\frac{1}{8} y_{3}+\frac{1}{2} y_{4}
$$

must hold. For all $x_{i}$ and $y_{i}$ we can obtain similar inequalities:

$$
\begin{aligned}
& 1 \leq \frac{1}{2}+x_{1}+\frac{1}{4} x_{2}+\frac{1}{4} x_{3}+\frac{1}{4} x_{4}+\frac{1}{2} y_{1}+\frac{1}{8} y_{2}+\frac{1}{8} y_{3}+\frac{1}{2} y_{4} \\
& 1 \leq \frac{1}{2}+\frac{1}{4} x_{1}+x_{2}+\frac{1}{4} x_{3}+\frac{1}{4} x_{4}+\frac{1}{2} y_{1}+\frac{1}{2} y_{2}+\frac{1}{8} y_{3}+\frac{1}{8} y_{4} \\
& 1 \leq \frac{1}{2}+\frac{1}{4} x_{1}+\frac{1}{4} x_{2}+x_{3}+\frac{1}{4} x_{4}+\frac{1}{8} y_{1}+\frac{1}{2} y_{2}+\frac{1}{2} y_{3}+\frac{1}{8} y_{4} \\
& 1 \leq \frac{1}{2}+\frac{1}{4} x_{1}+\frac{1}{4} x_{2}+\frac{1}{4} x_{3}+x_{4}+\frac{1}{8} y_{1}+\frac{1}{8} y_{2}+\frac{1}{2} y_{3}+\frac{1}{2} y_{4} \\
& 1 \leq \frac{1}{4}+\frac{1}{2} x_{1}+\frac{1}{2} x_{2}+\frac{1}{8} x_{3}+\frac{1}{8} x_{4}+y_{1}+\frac{1}{4} y_{2}+\frac{1}{16} y_{3}+\frac{1}{8} y_{4} \\
& 1 \leq \frac{1}{4}+\frac{1}{8} x_{1}+\frac{1}{2} x_{2}+\frac{1}{2} x_{3}+\frac{1}{8} x_{4}+\frac{1}{4} y_{1}+y_{2}+\frac{1}{4} y_{3}+\frac{1}{16} y_{4} \\
& 1 \leq \frac{1}{4}+\frac{1}{8} x_{1}+\frac{1}{8} x_{2}+\frac{1}{2} x_{3}+\frac{1}{2} x_{4}+\frac{1}{16} y_{1}+\frac{1}{4} y_{2}+y_{3}+\frac{1}{4} y_{4} \\
& 1 \leq \frac{1}{4}+\frac{1}{2} x_{1}+\frac{1}{8} x_{2}+\frac{1}{8} x_{3}+\frac{1}{2} x_{4}+\frac{1}{4} y_{1}+\frac{1}{16} y_{2}+\frac{1}{4} y_{3}+y_{4}
\end{aligned}
$$

The vertex $v$ contains 1 pebble, so the weights coming from other vertices will give excess weight at $v$. This excess weight is $\frac{1}{2}\left(x_{1}+x_{2}+x_{3}+x_{4}\right)+\frac{1}{4}\left(y_{1}+y_{2}+y_{3}+y_{4}\right)$. To determine the minimum value of the excess weight, so that the above inequalities are satisfied, a linear program can be solved. Using duality, one can easily verify that this minimum is $\frac{12}{25}$. (The minimum is taken when $x_{i}=0$ and $y_{i}=\frac{12}{25}$.)

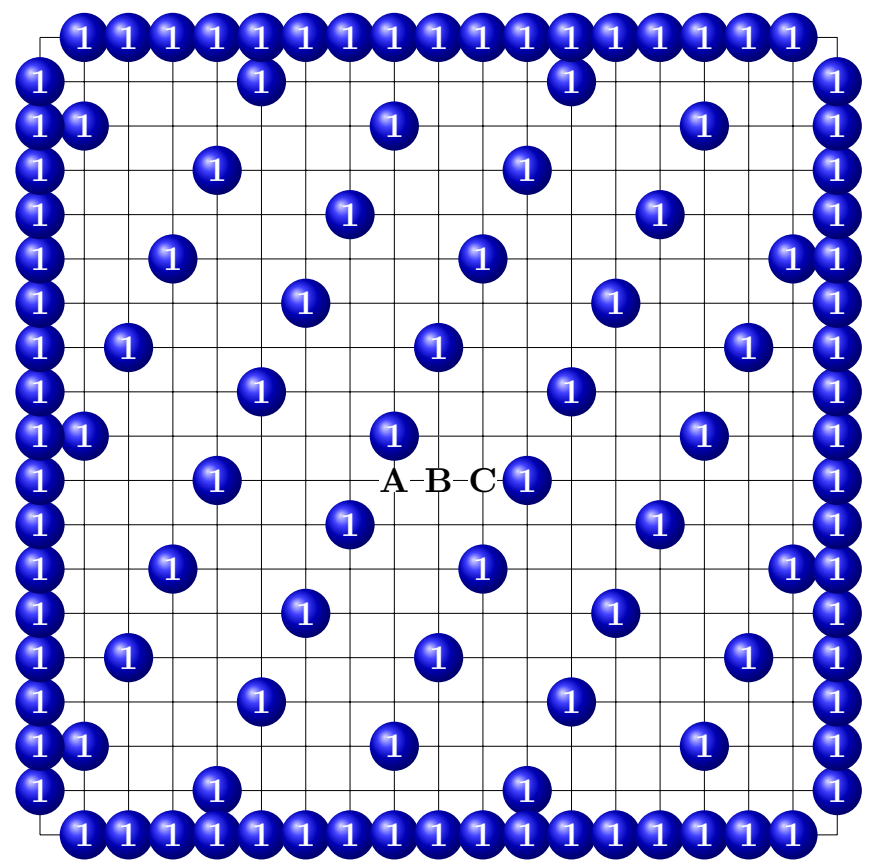

Fig. 5 Pebble distribution with $7-\varepsilon \leq \operatorname{IFCov}\left(G_{n \times n}\right)$

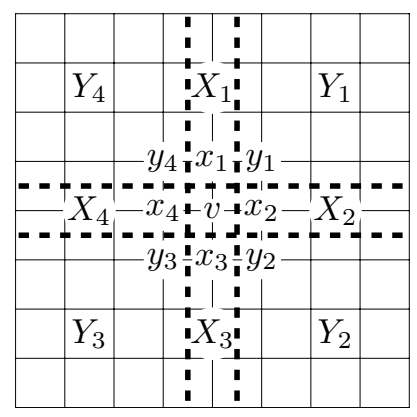

Fig. 6 Proof of the upper bound

Let $D$ be any pebble distribution. An easy calculation shows that the sum of the weight contributions of a single pebble to all other vertices on the grid is 9 , therefore the sum of weights for every vertex on the grid cannot exceed $9|D|$. If $D$ covers every vertex on the grid then the weight at every vertex is at least 1 , however, if the vertex contains $k$ pebbles then the excess weight on this vertex is at least $\frac{12}{25} k$ by Lemma 2. Hence $9|D| \geq n^{2}+\frac{12}{25}|D|$ holds. This implies that $\frac{n^{2}}{|D|} \leq 9-\frac{12}{25}=\frac{213}{25}=8.52$, proving our claim.

Theorem 2 implies that the best upper bound for the (integer) covering ratio we can hope using the approach of integer fractional covering is 7 . 


\section{Acknowledgement}

The first and second authors are partially supported by the National Research, Development and Innovation Office NKFIH (grant number K116769). The second and third authors are partially supported by the National Research, Development and Innovation Office NKFIH (grant number K108947).

\section{References}

[1] Györi, E., Katona, G. Y., Papp, L. F., Tompkins, C. "Lower bound on the optimal pebbling number of grids." manuscript

[2] Herscovici, D., Hester, B., Hurlbert, G. "Optimal Pebbling in Products of Graphs." Australian Journal of Combinatorics. 50, pp. 3-24. 2011.

URL: http://ajc.maths.uq.edu.au/pdf/50/ajc_v50_p003.pdf

[3] Hurlbert, G. "A survey of graph pebbling." In: Proceedings of the Thirtieth Southeastern International Conference on Combinatorics, Graph Theory, and Computing, Vol. 139, Boca Raton, FL, USA, 1999, pp. 41-64. URL: arXiv:math/0406024

[4] Hurlbert, G. "Recent progress in graph pebbling." In: Graph Theory Notes, N. Y., Vol. 49, pp. 25-37. 2005

URL: http://people.vcu.edu/ ghurlbert/papers/RPGP.pdf

[5] Hurlbert, G. "Graph pebbling." In: Handbook of Graph Theory. Gross, J. L., Yellen, J., Zhang, P. (eds.), Chapman and Hall/CRC, Kalamazoo, pp. 1428-1449. 2013.

https://doi.org/10.1201/b16132-83

[6] Xue, C., Yerger, C. "Optimal Pebbling on Grids." Graphs and Combinatorics. 32(3), pp. 1229-1247. 2015.

https://doi.org/10.1007/s00373-015-1615-5 